\title{
FIELD-SHAPE VARIATION INDUCED BY THERMAL CYCLING AND EXCITATION IN THE LHC DIPOLE
}

\begin{abstract}
O. Pagano, W. Scandale ${ }^{*}$, E. Todesco, C
\section{Abstract}

The field-shape imperfections measured at room temperature before and after thermal cycling and excitation up to maximum field in three $10 \mathrm{~m}$-long models of the LHC dipole are presented. A systematic variation is observed in the normal sextupole and decapole components. The variation of the other components turns out to be negligible or to randomly vary (as for normal and skew quadrupoles). We show how the systematic variation can be justified by assuming outward radial shift in four blocks of the inner coil. Qualitative tests using Fuji paper confirm this conjecture.
\end{abstract}

\section{INTRODUCTION}

The field quality in the LHC dipoles will depend on several effects, such as the nominal coil geometry, persistent currents, deformations induced by manufacturing and variation of the nominal geometry related to mechanical tolerances. When the magnet is powered at maximum current or when a quench occurs, coil movements may take place, and this may modify the field quality. In order to evaluate the relevance of this effect we present and analyse the experimental data relative to three dipoles models that have been measured before and after the first training cycle. Magnetic measurements were taken at room temperature and with low current $(\approx 20 A)$ : this assures that only the geometry of the coils affects the field quality. Therefore, variations of the field components are only induced by coil displacements. The aim of this work is the following: a) To evaluate which multipoles are affected by the first training cycle and how much; b) Separate the multipole variation that appears to be systematic in all magnets and apertures from the variations that are dependent on the magnet, on the aperture, and even on the position along the magnet axis; c) Evaluate what kind of coil displacement can produce these multipole variations. A similar analysis has been carried out in Ref. [1] for the IR quadrupoles of RHIC.

\section{EXPERIMENTAL DATA}

Three $10 \mathrm{~m}$ long dipoles models [2], named MBL1J2, MBL1N1 and MBL1N2 respectively, have been considered in our analysis. All of them have 5-block coils. The magnetic field inside both apertures were measured at room temperature using a $300 \mathrm{~mm}$-long $18 \mathrm{~mm}$-wide rotating coil and the standard multipolar expansion of the field was worked out. Normal and skew components was computed at a reference radius $R_{\text {ref }}$ of $17 \mathrm{~mm}$, along 11 longitudinal positions. We analysed the variation of the multipoles

\footnotetext{
*Email: walter.scandale@cern.ch
}

before and after the first training and power test (at $1.9 \mathrm{~K}$ and $12 k A$ ) for both apertures of the three models. Data for the dipole MB1LN1 are given in Fig. 1. Data for the dipoles MBL1N2 and MLB1J2 can be found in Ref. [3]. Variations averaged along the magnet axis that may be significant for the dynamics are summarised in Table 1. One can distinguish three groups of multipole variations:

- Systematic variations in all magnets and apertures $\left(b_{3}\right.$ and $b_{5}$ ), with a non-negligible longitudinal average.

- Random variations changing from magnet to magnet and from aperture to aperture $\left(a_{2}\right.$ and $\left.b_{2}\right)$, with a nonnegligible longitudinal average.

- Random variations that become small when averaged along the magnet axis: $\left(a_{4}, b_{4}\right.$ and $\left.b_{9}\right)$.

Table 1: Multipole variation induced by training cycle, averaged along the magnet axis, reference radius at $17 \mathrm{~mm}$, units of $10^{-4}$.

\begin{tabular}{|l|c|c|c|c|c|c|}
\hline & $\begin{array}{c}\text { J2 } \\
\text { left }\end{array}$ & $\begin{array}{c}\text { J2 } \\
\text { right }\end{array}$ & $\begin{array}{c}\mathrm{N} 1 \\
\text { left }\end{array}$ & $\begin{array}{c}\mathrm{N} 1 \\
\text { right }\end{array}$ & $\begin{array}{c}\mathrm{N} 2 \\
\text { left }\end{array}$ & $\begin{array}{c}\mathrm{N} 2 \\
\text { right }\end{array}$ \\
\hline$b_{3}$ & +1.29 & +1.36 & +1.63 & +1.65 & +1.07 & +0.68 \\
$b_{5}$ & -0.32 & -0.39 & -0.40 & -0.38 & -0.38 & -0.26 \\
$b_{2}$ & +0.25 & -0.64 & -0.13 & +0.06 & +0.35 & -0.47 \\
$a_{2}$ & +0.05 & -0.16 & -0.14 & +0.08 & -0.41 & -0.19 \\
$b_{4}$ & -0.02 & -0.02 & +0.02 & -0.05 & -0.05 & +0.02 \\
$a_{4}$ & -0.05 & +0.12 & +0.10 & -0.02 & +0.11 & +0.08 \\
$b_{9}$ & -0.08 & -0.04 & -0.04 & +0.03 & +0.10 & +0.12 \\
\hline
\end{tabular}

One can argue that only the multipole variations of the first and second type have an influence on particle motion in the LHC, since the longitudinal average along the magnet is large. Instead, the data of the third type are irrelevant for beam dynamics. Anyway, all the previous data are relevant for mechanics and will be used to analyse and predict the mechanical stability of the dipoles.

\section{ANALYSIS OF SYSTEMATIC EFFECT}

The data relative to the variation of $b_{3}$ and $b_{5}$, that appear to be systematic in all magnets and apertures, show that the ratio $\Delta b_{3} / \Delta b_{5}$ ranges from -2.7 to -4.5

Starting from the nominal geometry of the coil we computed the effect of simple movements of the blocks in order to work out a possible explanation for the systematic shift in $b_{3}$ and $b_{5}$. To this extent, we somehow arbitrarely only selected simple coil displacements, that preserve up-down and left-right symmetry:

- Displacement of a single block (and its corresponding symmetrical blocks) either by a radial shift or by a tangential shift or by a tilt, i.e. a rotation of the block around one 


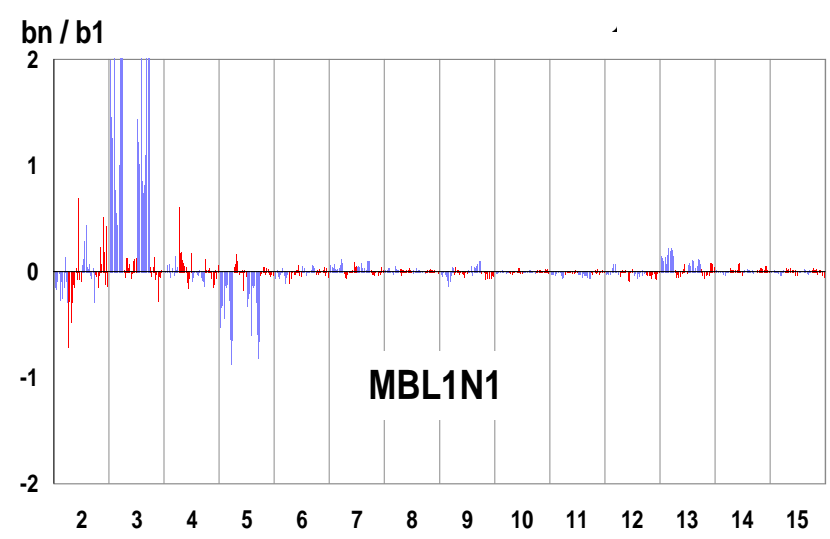

Figure 1: Multipole variation induced by training cycle in dipole MBL1N1; normal (black/blue) and skew (grey/red) components are measured in the two apertures and in 11 positions along the magnet axis. Above order 11, the amplitude of the harmonics is increased by a factor of 10 . Reference radius at $17 \mathrm{~mm}$, units $10^{-4}$.

vertex.

- Displacement of the inner layer or of the outer layer, either radial or tangential.

Amongst all these movements, the only one that features a ratio $b_{3} / b_{5}$ that falls into the measured range is a radial shift of block 5, 10, 15 and 20, as shown in Fig. 2. The amplitude of these shifts for each magnet and each aperture are given in Table 2.

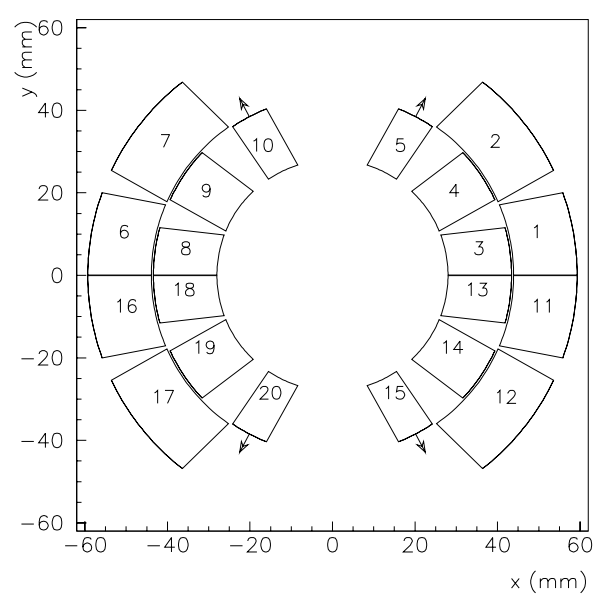

Figure 2: Nominal geometry of the 5-block coil; the arrows are the displacements (magnified by 100) needed to induce the observed $b_{3}$ and $b_{5}$ shifts

As it is pointed out in Ref. [4], the inverse problem is in general undetermined, i.e. the number of degrees of freedom relative to block displacements is larger than the number of experimental data to fit. Therefore, in general there exist $n$-parameter families of solutions that can fit experimental data, where $n$ is the difference between the number of degrees of freedom of the blocks and the numbers of multipole to fit. In our approach we arbitrarily reduce the complexity of the problem as follows: a) we reduce the number of multipole to fit by considering only $b_{3}$ and $b_{5}$ that produce the only systematic effect on all magnets and apertures; b) we reduce the number of degrees of freedom by considering only some simple movements of the blocks and by imposing a 4-fold symmetry.

Indeed, the validity of our solution is confirmed by additional experimental data provided by the Fuji papers, that provide a qualitative indication of the pressure existing between the collar and the coils in the different parts of the collar cavity. The analysis of Fuji papers inserted in a 5block $10 \mathrm{~m}$-long model shows that between the collars and the inner coils there is a reduced pressure during manufacturing, in all the four symmetric positions corresponding to blocks 5, 10, 15 and 20 [5]. This means that a gap is likely to occur between the collar and the coil during and after manufacturing, and that the effect of the Lorenz forces during the powering cycle is to push these blocks against the collar. The symmetry of the movements is caused by the symmetry of the electromagnetic forces and of the collar cavity.

Table 2: Amplitude (in $\mu m$ ) of outward radial movements of blocks 5, 10, 15 and 20 that produce observed shift in $b_{3}$ and $b_{5}$.

\begin{tabular}{|l|c|c|}
\hline Magnet & Left aperture & Right aperture \\
\hline MBL1J2 & 44 & 51 \\
MBL1N1 & 57 & 56 \\
MBL1N2 & 44 & 28 \\
\hline
\end{tabular}

\section{ANALYSIS OF THE RANDOM EFFECT}

For the analysis of the non-allowed harmonics we considered variation of multipoles in excess of $10^{-7}$ units, and the dependence along the magnet axis (no average is carried out over data). The most relevant components are $a_{2}$ and $b_{2}$. They can be generated by displacements that break the up-down symmetry and the left-right symmetry respectively. An asymmetric movement such as a positive radial displacement for blocks 5 and 10, and a negative one for blocks 15 and 20, would produce relevant multipole shifts in $a_{2}, a_{4}$, and $a_{6}$ only, and perfectly correlated. Indeed, such a correlation between even skew multipoles is not observed [3]. The same happens for even normal multipoles. The total loss of correlation between even multipoles implies that they are due to random displacements of the coils. These small displacements should also strongly depend on the position along the magnet axis, since as it is shown in Fig. 1 these multipoles have a very wide variation along the magnet axis.

In Ref. [3] we have shown through numerical simulations that a random displacement of blocks or of conduc- 
tors gives rise to very simple scaling laws for the multipoles: the $\sigma_{n}$, i.e. the standard devation of the distributions of normal or skew multipoles of order $n$ are approximately the same, and moreover they scale linearly with the amplitude of the random displacement $d$ and according to a power law in $n$ :

$$
\sigma_{n}(d)=d A B^{n} C^{n^{2}}
$$

with $A, B$ and $C$ independent of $d$. In Fig. 3 we compare the measurements of the multipoles along the magnet axis to the obtained scaling laws, with random displacements of the blocks, for the magnet MBL1N1. For each multipolar harmonics and for each aperture we have 11 values: we compute the sigma of these values of each harmonics measured in a dipole and we compare it with the corresponding sigma of the above equation.

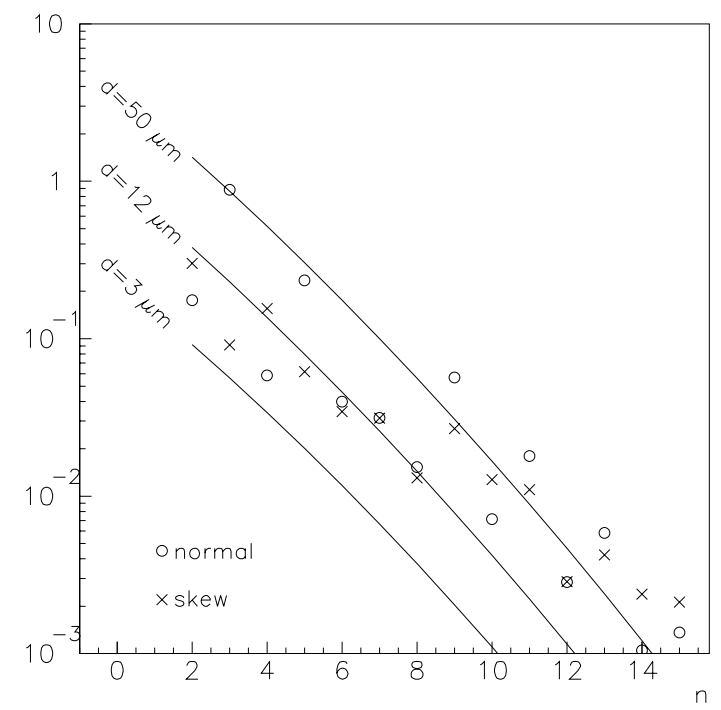

Figure 3: Multipoles of dipole MBL1N1 versus harmonic order $n$. Circles and crosses: r.m.s. of the multipoles computed for 22 values measured along the axis in both apertures, versus multipole order $n$. Solid lines: r.m.s multipoles obtained from scaling laws based on random displacements of blocks. Harmonics are given in $10^{-4}$ units at $17 \mathrm{~mm}$ reference radius.

Disregarding the peaks of the $b_{3}$ and $b_{5}$, related to the systematic effect described in section 3 , the other measured multipoles up to $b_{8}$ are consistent with random displacements of blocks with a sigma of around $12 \mu \mathrm{m}$ (see Fig. 3), or equivalently of random displacements of superconducting wire conductors whose sigma is around 25 $\mu m$ [3]. High order multipoles (from $b_{9}$ onward) seem in both cases to be larger compared to the scaling law. Anyway, these high order multipoles are rather low in absolute value and therefore should not endanger the particle dynamics. Similar results hold for the other magnets MBL1N2 and MBL1J2. One can observe that since the er- ror patterns are very similar, the three models feature the same behaviour, both from a qualitative and from a quantitative view point. Contrary to the effect on normal sextupoles and decapole discussed in the previous section, that should be easily corrected, the multipole shifts discussed in this section are very hard to cure, since they are induced by the displacement of several blocks, and since different displacements are observed in different transversal sections of the magnet.

\section{CONCLUSIONS}

The variation in the multipolar content of three $10 \mathrm{~m}$-long model LHC dipoles before and after training cycle is analysed. The systematic change of normal sextupole and decapole is explained in term of an outward radial shift of around $50 \mu \mathrm{m}$ of the four blocks $5,10,15,20$, symmetrically located in Fig. 2. The contact between these blocks and the collar is eventually too weak during manufacturing as confirmed by the analysis of the Fuji paper inserted in one of the long models. This effect could be cured by guaranteeing a complete contact between collars and inner coil during collaring. We have already indications that 6-block coil design and an improved collaring procedure at an advanced state of specification are sufficient to fulfil this requirement.

The changes of the other multipoles are interpreted in terms of a random displacement of all blocks. Experimental data are compatible with simulations where the random displacement of the block has an r.m.s. value of 12 $\mu m$. This provides an useful order of magnitude of the coil movements that can be expected during the first powering cycle.

Finally, we remark that all the three models show very similar features, both for the systematic and for the random effects. In fact, in terms of field quality it seems that the three analysed magnets are equivalent.

We would like to thank A. Devred, D. Giloteaux, R. Gupta, D. Leroy, M. Modena. D. Perini, S. Russenschuck, G. Spigo, D. Tommasini, J. Vlogaert, and C. Wyss.

\section{REFERENCES}

[1] R.Gupta et al. These Proceedings.

[2] J. Billan et al., EPAC 99, (1999) 2041-3.

[3] O. Pagano, W. Scandale,E. Todesco, LHC Project Note 180 (1999).

[4] S. Russenschuck, LHC Project Report 159 (1997).

[5] M. Modena, private communication (1999). 